\section{Immunology 101: The Basics of Immunoglobulins and Immunostaining}

\author{
W. Gray (Jay) Jerome \\ Vanderbilt University Medical Center \\ jay.jerome@mcmail.vanderbilt.edu
}

The ability to immunologically link microscopic tags to specific proteins has produced major advances in all forms of microscopy. Initially researchers needed to make and label their own antibodies. This required a good working knowledge of immunology. Today labeled anitbodies are readily available from a variety of vendors. Although this has greatly facilitated the expansion of immunolabeling techniques, it has also led to their use by researchers with little or no background in immunology. While this is not a problem for simple, straightforward situations, the quality of data produced in more specialized experiments can suffer. Being an expert in immunology is not necessary for the average user of immunohistochemistry techniques, but some basic understanding of what antibodies are, how they are formed, and how best to use them as a cellular probe can greatly enhance the results of "non-routine" staining procedures. In this regard it is worth reviewing some basic immunology principles for the naive user and sharing some insights with the more experienced user. This is the general goal of this brief note. Specifically, we will review what antibodies are, how they are formed, the differences between immune serum and purified immunoglobulins, and the differences between polyclonal and monoclonal antibodies. Following that we will discuss the pros and cons of various preparative techniques, including types of fixation, types of permeabilization, and the various immunoprobes from fluorophores to gold labels.

Immunoglobulins, particularly immunoglobulin $\mathrm{G}(\mathrm{IgG})$, are the basic tool of immunohisto/cytochemistry. An antibody is an immunoglobulin molecule. It is produced by cells of the immune system in response to a foreign challenge (antigen). A good antibody binds to its antigen specifically and with high affinity. This binding is exploited to tether a microscopically visible marker to the antigen. In this way, the molecule can be specifically localized within cells or tissue. To produce superior localization without artefact, a good antibody is required. A little time spent obtaining and testing antibodies to insure they are useful in immunostaining will save the investigator a lot of time and trouble later.

There are a variety of paradigms for immunostaining. The most basic procedure is to label the antibody directly with a microscopic marker. This simplifies the staining procedures but interactions between the label and the immunoglobulin often lessens the usefulness of the antibody as a histochemical reagent. The most common method is the two-step or indirect method. In this method the antibody is reacted with the sample and allowed to bind to the antigen. Then the preparation is incubated with a second antibody. This antibody contains the label and has been carefully selected because its properties are not adversely affected by the tag and because of its specificity for binding to the first antibody. For instance, to localize human acid phosphatase enzyme an IgG antibody can be raised in a rabbit that recognizes human acid phosphatase. After tethering the anti-acid phophatase to the acid phosphatase molecules in the cell, the anti-acid phophasphatase can be localized by tethering a labeled goat IgG that specifically binds all rabbit IgGs but does not bind human IgG. These general labeled secondary antibodies are readily available commercially. Although this twostep method takes a little more time, it saves the researcher the time of making multiple primary antibodies, labeling each antibody, and testing each for specificity after labeling in order to find one that is useful. Variations on this theme include exploiting the ease of biotinylating antibodies and the strong affinity of biotin for avidin as a means of localizing an antigen. Since each avidin will bind four biotins, modifications of the technique can be used to amplify the labeling of an antigen that occurs in low abundance.

Several critical factors go into the decision of what method is used in an immunolocalization. These include how cell integrity is to be maintained, how the antigen is going to be made accessible to the antibody, what type of tag will be used, and how the localization data will be acquired and analyzed. For instance, are the cells to be fixed and, if so, what fixative should be employed? Do the cells need to be permeabilized to allow the antibody access to the antigen or is the antigen readily accessible? Do you need to visualize multiple antigens at the same time? Do you need the resolution of an electron microscope or is light microscopy sufficient? All of these are considerations in choosing a methodology. In the end, some experimentation is often required to work out optimum techniques and a working knowledge of immunology is extremely useful in determining what changes to make.

NOTE: This very brief introductory note draws from a Technologists' Forum presentation at the 2002 M\&M Meeting in Quebec. See author abstract in the M\&M 2002 Procedings. ...Editor

\section{Position Available
Transmission Electron Microscopist}

Princeton University's Department of Molecular Biology is seeking a Transmission Electron Microscopist to run a new LEO912AB. Duties will include: (1) teaching and training undergraduate and graduate students, post-docs and faculty on instrument operation, (2) preparation and sectioning of diverse biological specimens including yeast, virally infected mammalian cells, brain slices and Drosophila embryos, using a variety of methods including high-pressure freezing and freeze substitution techniques, (3) daily microscope system checks and scheduling of users, (4) general lab maintenance and supply. The candidate should have a BS/MS in a biological field and several years of TEM experience. Knowledge of ultra-structural and immuno-labeling protocols, the operation and care of microtomes and other equipment needed for specimen preparation, a basic understanding of digital imaging and the ability to manage and administer digital workstations and image archives is essential. The candidate must be highly interactive, willing to collaborate on diverse projects and able to identify and research the best methods of specimen preparation and examination. Rank and salary are dependent upon qualifications and experience. Please send curriculum vitae, a list of references and representative samples of your work to Professor Mark Rose, Chair, Search Committee, Department of Molecular Biology, Princeton University, Princeton, NJ 08544-1014.

Princeton University is an Equal Opportunity/Affirmative Action Employer: 


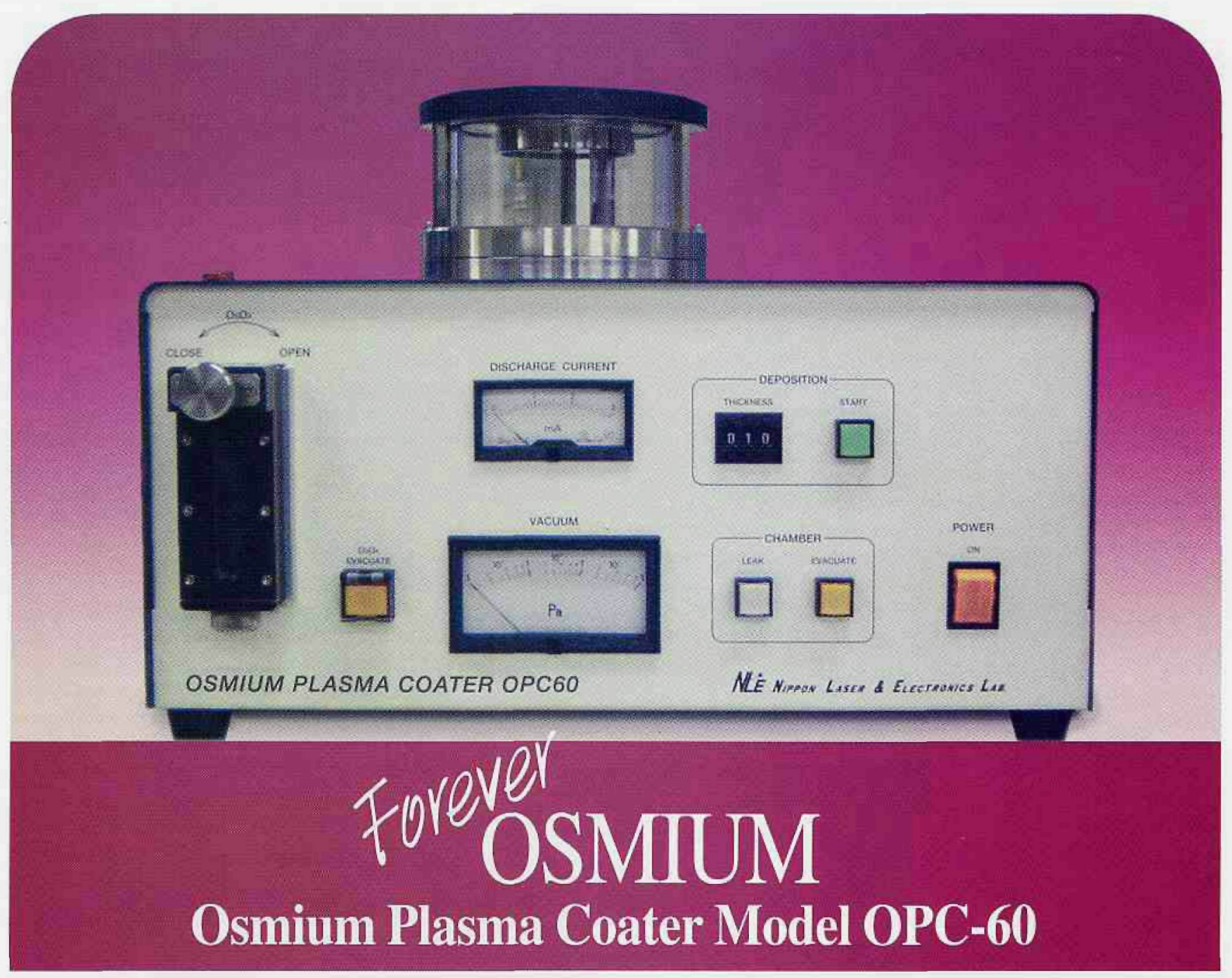

The ultimate coating for SEM samples. The OPC-60 Osmium Plasma Coater is the result of many years of careful research at Nippon Laser and Electronics Lab (NL\&EL) in Nagoya, Japan.

It was a collaboration of NL\&EL, experienced SEM researchers at several of the leading universities in Japan and some of the world's leading manufacturers of SEM and FESEMs, with headquarters in Japan. The result is the ultimate coating device for coating SEM samples and especially FESEMs

- Finest particle size available in a coating, giving greater definition to samples at the highest magnifications, thereby outperforming gold or chromium.

- Fast turnaround time between samples and a longer shelf life.
- Less long-term maintenance costs.

- Easy set-up time and completely safe when used as directed.

Visit www.2spi.com to see why the OPC-60 will be "forever" your favorite sample coater.

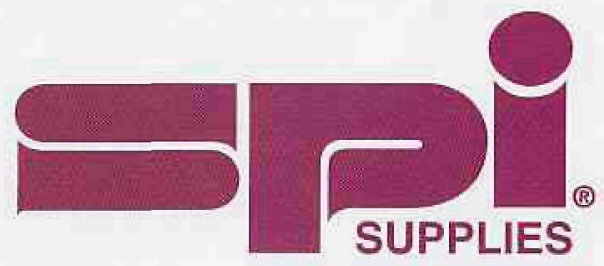

\section{SPI Supplies Division of STRUCTURE PROBE, Inc.}

P.0. Box 656 - West Chester, PA 19381-0656 USA

Phone: 1-610-436-5400 • 1-800-2424-SPI (USA and Canada) • Fax: 1-610-436-5755 • E-mail: sales@2spi.com 